\title{
Characterization of Nanomaterials with Total Scattering and Pair Distribution Function Analysis: Examples from Metal Oxide Nanochemistry
}

\author{
Kirsten M. Ø. Jensen ${ }^{\star}$
}

\begin{abstract}
The development of new functional nanomaterials builds on an understanding of the intricate relation between material structure and properties. Only by knowing the atomic arrangement can the mechanisms responsible for material properties be elucidated and new materials and technologies developed. Nanomaterials challenge the crystallographic techniques often used for structure characterization, and the structure of many nanomaterials are therefore often assumed to be 'cut-outs' of the corresponding bulk material. Here, I will discuss how Pair Distribution Function (PDF) analysis of total scattering data can aid nanochemists in obtaining a structural understanding of nanoscale materials, focusing on examples from metal oxide chemistry.
\end{abstract}

Keywords: Metal oxide nanoparticles · Pair Distribution Function analysis · Structure analysis · X-ray scattering

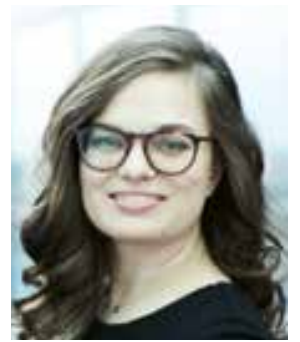

Kirsten M. Ø. Jensen is an associate professor at the Department of Chemistry at University of Copenhagen. She received her PhD in Chemistry from Aarhus University in 2013. She started her research group at University of Copenhagen in 2015, after a postdoctoral fellowship at Columbia University. Her research concerns nanomaterials, focusing especially on characterization of atomic structure using X-ray scattering techniques.

\section{Introduction}

Understanding the relation between material synthesis, structure and properties is the very core of materials chemistry, and discoveries of new materials and applications rely on being able to determine and characterize atomic structure. Over the past century, crystallography has played an enormous role in this work. The first question a chemist asks after a synthesis is "what is it?", and crystallography and diffraction techniques are here indispensable. Powder diffraction especially is essential for material characterization, and PXRD experiments and Rietveld refinement are core tools in a material chemists' toolbox. ${ }^{[1]}$ However, the intense focus on nanoscience in all fields of materials chemistry challenges the crystallographic methods commonly used. ${ }^{[2]}$ Bragg diffraction and crystallographic techniques rely on the existence of long-range atomic order, and when nanoparticles get small, this assumption breaks down. This is illustrated in Fig. 1A, where calculated diffraction patterns from iron oxide nanocrystals of different sizes are shown. When the particles are smaller than $5 \mathrm{~nm}$, the broadening of Bragg peaks makes it difficult to extract detailed structural information. Any nanosize-induced structural changes to the particle structure will complicate this analysis much further, as the as-

${ }^{*}$ Correspondence: Dr. K. M. Ø. Jensen, E-mail: kirsten@chem.ku.dk Department of Chemistry, University of Copenhagen, Denmark sumption of a small unit cell repeating over and over in 3D may not be appropriate for materials with dimensions on this length scale. This hinders mapping of the relation between structure and properties in nanostructured materials and is a bottleneck in nanomaterials development that many chemists face.

Pair Distribution Function (PDF) analysis of X-ray or neutron total scattering data is an excellent method for characterizing such nanoscale structures. A total scattering experiment is very similar in nature to a conventional powder diffraction experiment, except data are collected over a wider Q-range, as will be discussed below. The PDF is the Fourier transformation of the total scattering data, and represents a histogram of all interatomic distances in the sample, regardless if the sample is crystalline, nanostructured, or amorphous. The history of PDF analysis goes back to early works of Debye in $1916^{[3]}$ and Prinz and Zernike in $1927,{ }^{[4]}$ and the first X-ray PDF experiments were done in the 1930s. ${ }^{[5]}$ For several decades PDF was often used as a last resort for structural characterization of materials with limited order such as liquids and glasses, as well as amorphous materials, as seen in Rosalind Franklin's work on disordered carbons. ${ }^{[6]}$ Limitations in instrumentation, as well as the need for cumbersome and computationally heavy calculations held back the use of and interest in PDF. The field changed significantly with the development of high energy neutron sources and synchrotron beamlines with high energy X-ray radiation, as well as with developments of detector technology allowing efficient and fast detection of X-rays. The potential of PDF for local structure analysis of crystalline materials was marked by seminal work by Billinge and Egami in the early 1990s. ${ }^{[7]}$ Since then, the use of PDF for materials structure analysis has continued to grow through the development of user-friendly software, dedicated synchrotron beamlines and neutron instruments for PDF, textbooks, reviews and tutorials, along with an increased interest in short-range order of materials. PDF has thus over the last decades developed from being an exotic, rarely used technique to an important characterization method in physics, chemistry, and beyond. ${ }^{[8]}$ Today, PDF analysis plays an increasing role in nanomaterial characterization. For 'large' nanoparticles, $e$.g. with dimensions above 8-10 nm, a simple Q-space Rietveld refinement 

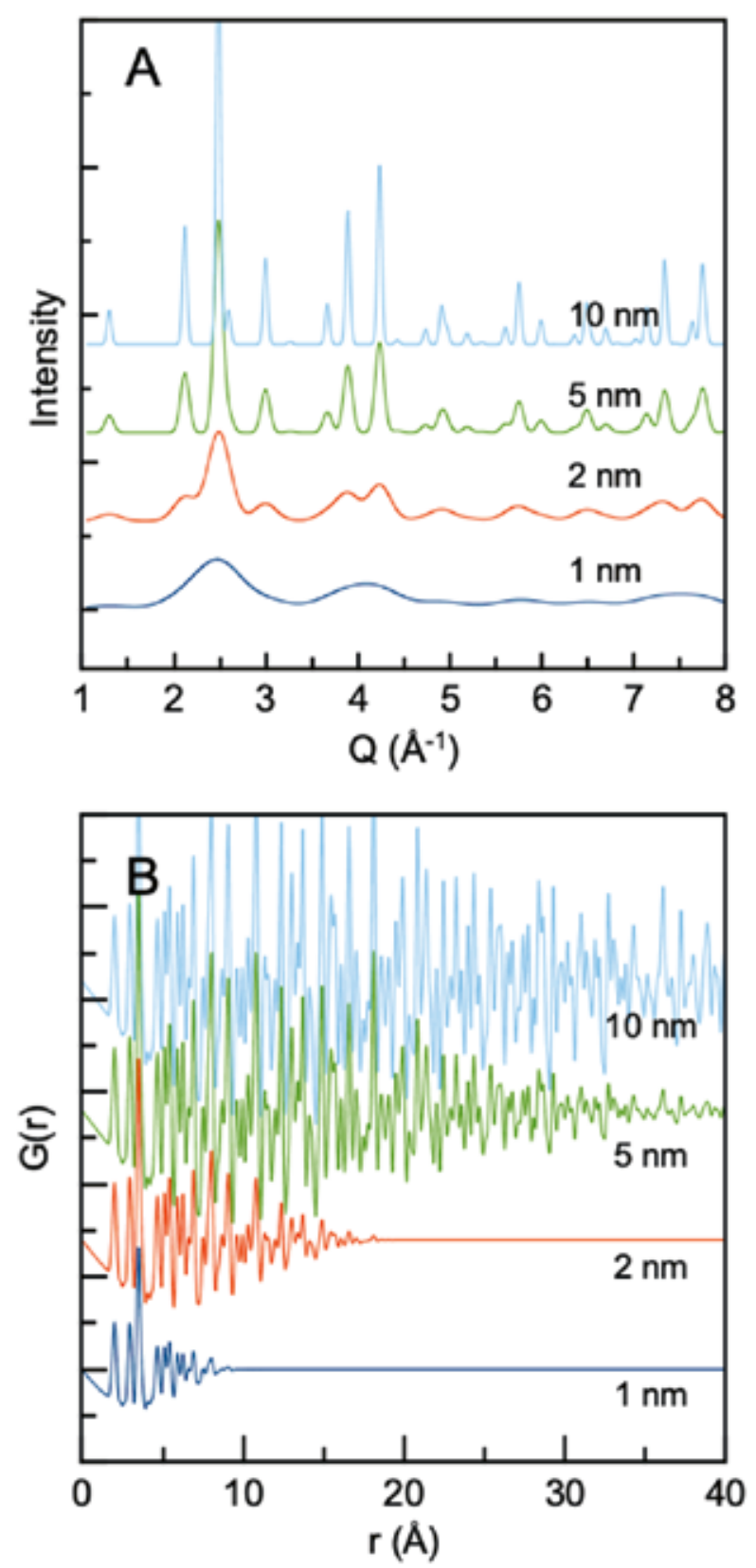

Fig. 1. Calculated (A) PXRD patterns and PDFs (B) from $\mathrm{Fe}_{3} \mathrm{O}_{4}$ nanoparticles of sizes $1 \mathrm{~nm}, 2 \mathrm{~nm}, 5 \mathrm{~nm}$ and $10 \mathrm{~nm}$. The data are calculated assuming a perfect $f c c$ structure.

is usually a good approach for structure characterization. Below this range, the features in the scattering pattern become broad, and structural rearrangements may happen, which results in diffuse scattering. It is thus when particles get very small or disordered, and when these size-dependent effects appear, that PDF comes into its right as a very powerful technique for structure analysis.

In this short review, I will illustrate how the PDF technique can be used by chemists working with nanomaterials for structural characterization. The PDF literature is vast, and I will here only discuss a few, selected examples that illustrate different aspects of PDF analysis. I will focus on metal oxide nanostructures, as metal oxide chemistry is becoming more and more important for e.g. battery materials, catalysis and beyond. I will show examples of both size-dependent atomic structure in nanoparticles, structure analysis of amorphous oxides, and studies of nanoparticle formation and growth. A more extensive review of PDF for nanomaterials is given in e.g. ref. [8b], and other applications of PDF, as well as PDF theory, have been described in detail in textbooks ${ }^{[9]}$ and recent reviews. ${ }^{[2,8 b, 10]}$

\section{The Pair Distribution Function}

A PDF is obtained by Fourier transforming total scattering intensities, most often obtained using X-rays or neutrons. The Fourier transform is done over the structure function $\mathrm{S}(\mathrm{Q})$, as here expressed for X-ray scattering:

$$
\begin{aligned}
& S(Q)=\frac{I_{c}(Q)-\left\langle f(Q)^{2}\right\rangle+\langle f(Q)\rangle^{2}}{\langle f(Q)\rangle^{2}} \\
& G(r)=\left(\frac{2}{\pi}\right) \int_{Q_{\min }}^{Q_{\max }} Q(S(Q)-1) \sin (Q r) d Q
\end{aligned}
$$

$\mathrm{S}(\mathrm{Q})$ is obtained from $\mathrm{I}_{c}(\mathrm{Q})$, which is the experimentally determined coherent scattering intensity. $f(Q)$ is the atomic form factor for the elements in the sample, and Q is the momentum transfer, related to the scattering angle and the X-ray wavelength through $Q=\frac{4 \pi \sin (\theta)}{\lambda}$. Over the last decades, several user-friendly programs ${ }^{[11]}$ have been developed, which can be used to obtain $\mathrm{I}_{\mathrm{c}}(\mathrm{Q}), \mathrm{S}(\mathrm{Q})$, and $\mathrm{G}(\mathrm{r})$ from experimental X-ray or neutron total scattering data, making PDF analysis accessible to a broad range of scientists.

The Q-range included in the Fourier transformation, i.e. the $\mathrm{Q}_{\min }$ and $\mathrm{Q}_{\max }$ values, determine the r-resolution in the resulting PDF, and therefore the amount of information and detail that can be extracted. In order to obtain atomic scale structural information, a $Q_{\max }$ value of at least $15-20 \AA^{-1}$ is needed, and often, an even higher value is used. This means that radiation with a short wavelength, i.e. high energy, is required. A high flux of X-rays or neutrons is also essential, as good data statistics are needed to minimize noise in the final PDF. In the case of X-rays, PDF experiments are therefore often done at synchrotron sources with dedicated beamlines for total scattering. In recent years, laboratory instruments with $\mathrm{Ag}$ or Mo X-ray tubes, suitable for PDF analysis, have become increasingly common as the interest for PDF analysis grows. Total scattering measurements can also be performed using neutrons or electrons, ${ }^{[12]}$ and a PDF can thus be obtained using a transmission electron microscope, available in many material chemistry laboratories. This 'ePDF' method is expected to become more commonly applied in the coming years. ${ }^{[13]}$

The PDF represents a histogram of interatomic distances in the sample. The G(r) function, which is obtained directly from the Fourier transform of $\mathrm{S}(\mathrm{Q})$, is the reduced par distribution function. $\mathrm{G}(\mathrm{r})$ is closely related to the $\mathrm{R}(\mathrm{r})$ function, the radial distribution function:

$$
\begin{aligned}
& G(r)=\frac{R(r)}{r}-4 \pi r \rho_{o} \\
& R(r)=\sum_{v} \sum_{\mu} \frac{f_{\mathrm{v}} f_{\mathrm{u}}}{\langle f\rangle^{2}} \delta\left(r-r_{v u}\right)
\end{aligned}
$$

The $\mathrm{R}(\mathrm{r})$ is fairly intuitive: Two atoms, $v$ and $u$, separated by a distance $r_{v,}$, will give rise to a delta function in the $\mathrm{R}(\mathrm{r})$ function at this r-value, and the scattering power of the two atoms determine the intensity of the peak. The full $R(r)$ is obtained by summing over peaks from all atomic pairs in the sample. While the $R(r)$ function increases with increasing $r$, the G(r) function oscillates around zero in the limit of large $r$ values, and the intensity of G(r) peaks represent the probability of finding a pair of atoms with a separation of $r$, compared to the average atomic number density in the sample.[14]

Calculated PDFs (G(r) functions) from iron oxide nanoparticles of different sizes are given in Fig. 1B. As can be seen, infor- 
mation on both atomic structure and crystallite size can be directly extracted from the PDF. This intuitive nature of the PDF means that much information can be obtained from a model-free analysis. However, through PDF modelling, much more, and more reliable, information can be obtained. PDFs can easily be calculated from atomic structure models, e.g. a crystal structure or a model of a discrete object such as molecule-like clusters or nanoparticles. These calculated PDFs can then be compared with experimental data, and the parameters in the models can be refined to fit to the data. Several strategies for PDF modelling exist, where more or less complex models can be used to describe the data. The 'realspace Rietveld' method, ${ }^{[15]}$ where the modelling is based on a periodic crystal structure model is very commonly used, especially for nanomaterial analysis as discussed here. PDF modelling based on discrete structure models can be done using the Debye equation, and the PDF from amorphous and highly disordered materials can be analyzed through e.g. 'big-box' modelling using the Reverse Monte Carlo method. ${ }^{[10]}$ The most suitable method for data analysis will be dependent on the problem at hand, as well as the data obtained in the experiment.

\section{Size-dependent Structure in Metal Oxide Nanoparticles}

The atomic structure of metal oxides is often described in terms of $\left[\mathrm{MO}_{\mathrm{x}}\right]$ polyhedra, where $\mathrm{M}$ is a metal. By assembly of e.g. $\left[\mathrm{MO}_{6}\right]$ octahedra and $\left[\mathrm{MO}_{4}\right]$ tetrahedra through edge-, corner, or face-sharing, a plethora of crystal structures appears, and this vast structural space and possibility for crystal engineering means that the list of applications of metal oxides is almost endless. However, nanosizing of metal oxides can provide even more options, as it is well known that structural changes can take place when going to the nanoscale. For example, size-induced defects can appear, which can heavily influence material properties. To take advantage of metal oxide nanoparticles, it is therefore crucial that we can map the size/structure relations in metal oxide nanomaterials.

PDF is an excellent technique for studies of these effects. For example, we have used 'real-space Rietveld' analysis for studies of the defect chemistry of molybdenum oxides; ${ }^{[16]}$ an important material for catalysis and a potential battery material. $\mathrm{MoO}_{2}$ normally crystallizes in a distorted rutile structure, however, significant structural changes take place when going to the nanoscale, which affect the material properties. ${ }^{[17]} \mathrm{We}$ studied these effects with PDF in $\mathrm{MoO}_{2}$ nanoparticles of two different sizes; $4 \mathrm{~nm}$, and $40 \mathrm{~nm}$, as well as in bulk $\mathrm{MoO}_{2}$. PDF analysis of the three samples (Fig. 2A-C) showed that the expected distorted rutile structure gave a good fit to the PDF from the bulk $\mathrm{MoO}_{2}$ sample (Fig. 2A). However, the model only describes the average structure of the crystalline, $40 \mathrm{~nm}$ $\mathrm{MoO}_{2}$ particles (i.e. the r-range above $5 \AA$ in the PDF, Fig. 2B). The distorted rutile model failed to fit the PDF peak arising from Mo-Mo distances in edge-sharing $\left[\mathrm{MoO}_{6}\right]$ octahedra at $3.8 \AA$. From the fit and the difference curve, it is clear that the crystalline sample contains more edge-sharing octahedra than accounted for in the rutile model, and further PDF analysis showed that this misfit arose from uncorrelated point defects in the rutile structure, where additional edge-sharing octahedra appear.

For the $4 \mathrm{~nm}$ nanoparticles, a misfit is seen over the full range of the PDF (Fig. 2C). By comparing the model and the data, it is clear that even more edge-sharing $\left[\mathrm{MoO}_{6}\right]$ motifs appear than in the larger crystalline nanoparticles, and this affects also the longer-range structure. By considering defects in known rutile systems, we found that a structure model based on the presence of crystallographic shear planes, ${ }^{[18]}$ could describe the observed PDF. A very simplified structure model was constructed by building an interwoven rutile model, which reflects the appearance of shear-planes. As illustrated in Fig. $3 \mathrm{~B}$, an 'extra' rutile structure was incorporated into the lattice by adding a new Mo atom in an interstitial, partially occupied site in the unit cell. The PDF analysis was complemented by HR-TEM which agreed with the proposed model. As seen in Fig. 3C, the TEM images showed that Mo atoms can indeed be found in interstitial sites, as expected from the PDF analysis. This study of molybdenum oxides show how defect chemistry known from bulk structures can completely dominate in nanoparticles and thus affect material properties. The use of PDF and simple 'real-space' Rietveld analysis allowed an intuitive identification of the defects, which led to the development of new models. Defects and size-dependent structure have been

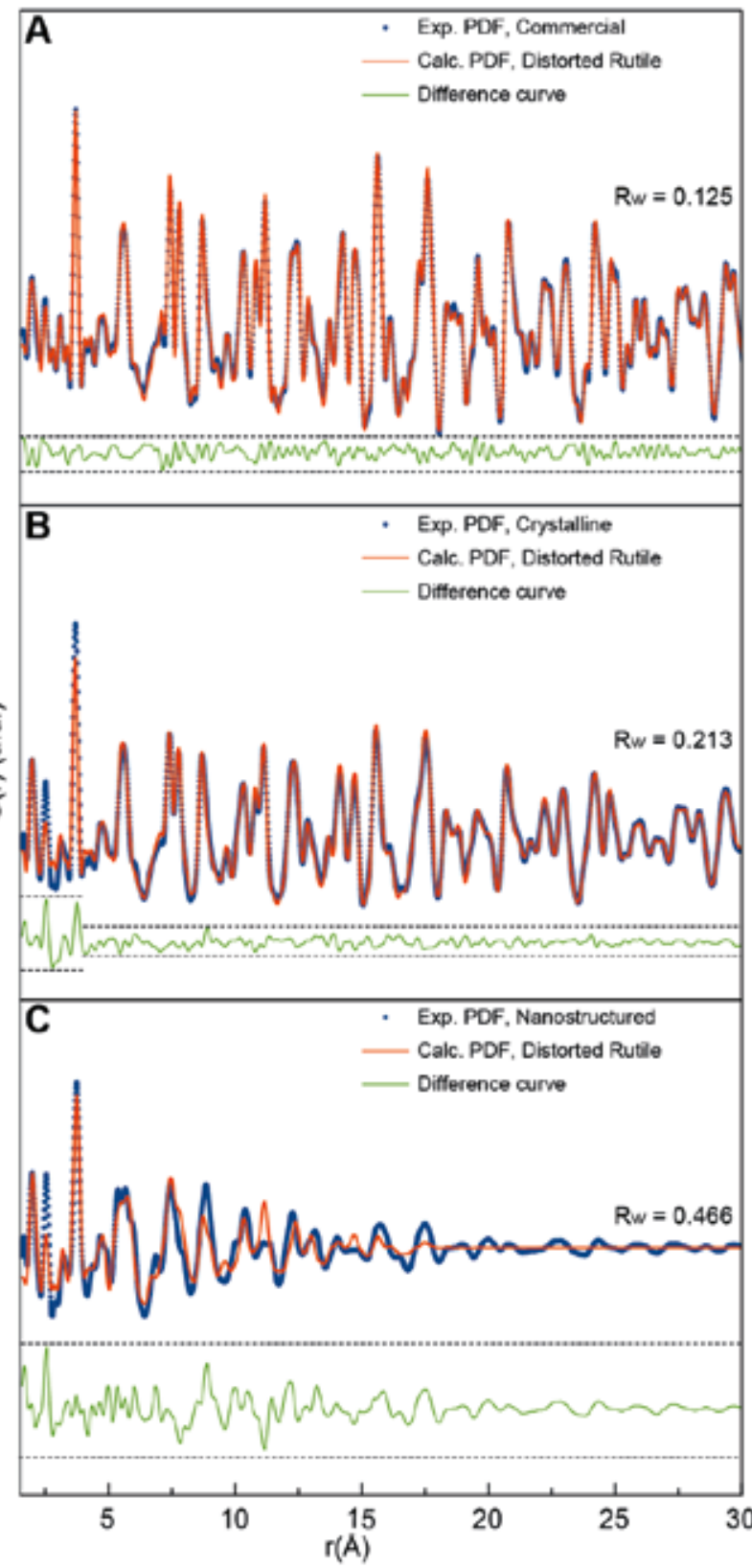

Fig. 2. Fits to the PDFs of A) a bulk $\mathrm{MoO}_{2}$ sample, B) crystalline nanoparticles, ca. $40 \mathrm{~nm}$ and $\mathrm{C}$ ) nanostructured samples, ca. $4 \mathrm{~nm}$ using the $\mathrm{MoO}_{2}$ rutile model. The model gives a good description of the PDF from the bulk sample, while it only fits the long-range order in the $40 \mathrm{~nm}$ nanoparticles. The model gives a poor fit of the entire PDF range for the small $(4 \mathrm{~nm})$ nanoparticles. Reprinted with permission from T. L. Christiansen, E. D. Bøjesen, M. Juelsholt, J. Etheridge, K. M. Ø. Jensen, ACS Nano 2019, 13, 8725-8735. Copyright 2019 American Chemical Society. 

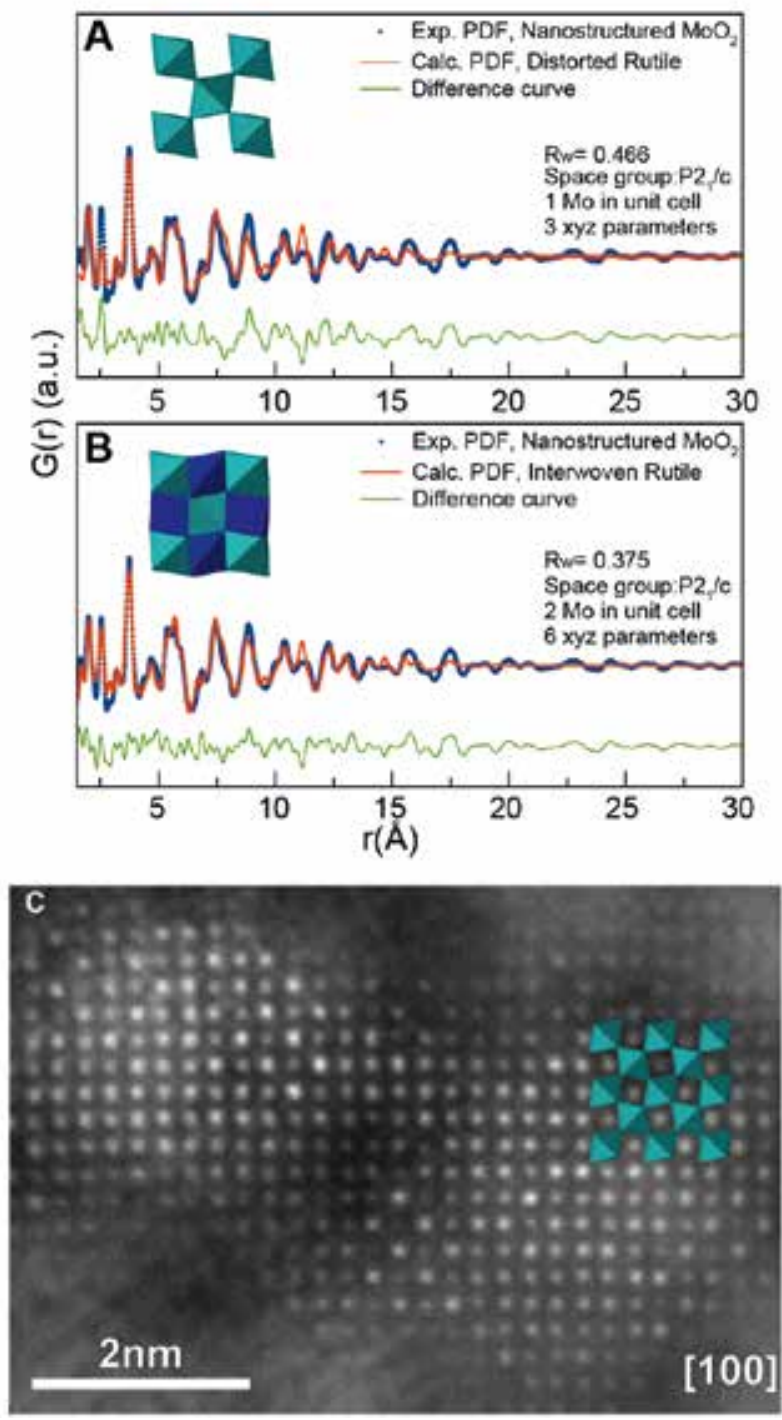

Fig. 3. Fits to the PDF obtained from nanostructured $\mathrm{MoO}_{2}$ using two different models. (A) The bulk rutile structure and (B) a 'double' rutile model representing disordered shear-planes. (C) High-resolution TEM image from the sample. A rutile structure model is overlaid on the image. Reprinted with permission from T. L. Christiansen, E. D. Bøjesen, M. Juelsholt, J. Etheridge, K. M. Ø. Jensen, ACS Nano 2019, 13, 87258735. Copyright 2019 American Chemical Society.

characterized in a range of different metal oxide systems, including $\mathrm{Al}_{2} \mathrm{O}_{3},{ }^{[19]} \mathrm{TiO}_{2},{ }^{[20]}$ iron oxides, ${ }^{[21]}$ and ferrihydrite. ${ }^{[22]}$

\section{Amorphous and Disordered Metal Oxides}

PDF also gives the opportunity to study highly disordered oxide materials, which have recently received much attention for applications in electrocatalysis. ${ }^{[23]}$ For example, Du et al. used PDF to establish the structure of an amorphous, or highly disordered, cobalt oxide for water splitting, giving rise to only diffuse scattering in the scattering pattern. ${ }^{[24]}$ From Extended X-ray Absorption Fine Structure (XAFS) analysis of the material, a cubane-type motif built up of edge-sharing $\left[\mathrm{CoO}_{6}\right]$ octahedra was expected. ${ }^{[25]}$ However, EXAFS only provides structural information on the very local atomic range, and PDF was therefore needed to characterize the longer range atomic structure, and to understand the arrangement of $\left[\mathrm{CoO}_{6}\right]$ octahedra. A range of different models of cobalt oxide models were built, where both the domain size and atomic arrangements were varied. PDFs were then calculated from the models using the Debye equation and compared to the experimental PDFs. The best fitting model was a structure with $13\left[\mathrm{CoO}_{6}\right]$ motifs arranged with edge-sharing, as cut directly from a LiCoO ${ }_{2}$ crystal structure. The comparison between the simulated and experimental PDFs is shown in Fig. 4, and the structure clearly describes the experimental PDF peaks well. The model was improved through small refinements of the atomic structure, thus allowing to map the material structure/ property relations in much more detail.

Many layered oxide materials show a large degree of interand intralayer disorder, which can affect their properties for $e . g$. intercalation reactions in electrochemical cells. ${ }^{[26]}$ Such disorder has been extensively studied with PDF.[27] For example, Batchellor et al..$^{[28]}$ have used PDF to characterize the structure of nanocrystalline layered nickel iron oxyhydroxides, consisting of layers built up from edge-sharing $\left[\mathrm{MO}_{6}\right]$. These materials are highly active electrocatalysts, but disorder in the layering have made it difficult to understand the relation between material preparation, structure and properties, and many questions remain unanswered. Using PDF, Batchellor et al. were able to characterize both the local structure, the layer disorder, and the domain size in the material, which was dependent on both synthesis method and catalytic use.

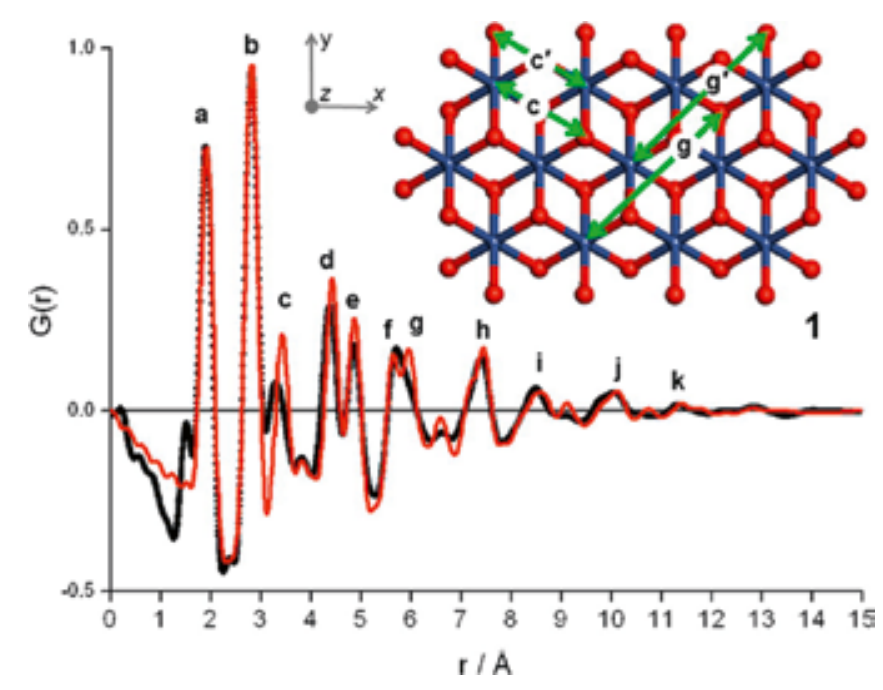

Fig. 4. Fit to an experimental X-ray PDF obtained from nanostructured cobalt oxide. The mode shown in the insert was obtained by cutting out a motif from the $\mathrm{LiCOO}_{2}$ crystal structure. Reprinted with permission from Du et al., J. Am. Chem. Soc. 2012, 134, 11096-11099. Copyright 2012 American Chemical Society

\section{d-PDF: Structural Changes upon Adsorption}

Using 'differential PDF' (d-PDF), it is possible to resolve small structural changes in materials after e.g. a chemical reaction. For example, Chupas et al. used d-PDF to determine the nature of the catalytic surface sites in $\mathrm{Al}_{2} \mathrm{O}_{3}$ nanoparticles by adsorbing a basic probe molecule (monomethylamine, MMA), and identifying its coordination to the nanoparticle surface.[29] Experimental PDFs from $\gamma-\mathrm{Al}_{2} \mathrm{O}_{3}$ nanoparticles both with and without adsorbed MMA were obtained and subtracted from each other, giving the differential PDF plotted in Fig. 5, where clear peaks relating to the interaction between the molecule and surface could be identified. Analysis of the d-PDF showed that the active sites are five-coordinate, as MMA coordinates directly to an $\mathrm{Al}$ surface atom.

d-PDF studies are also widely used for studies of catalysts, where nanoparticles are often dispersed on high-surface area supports in order to save active material and prevent agglomeration. Structural analysis of such materials can be using the d-PDF method, ${ }^{[30]}$ where data from the support both with and without 


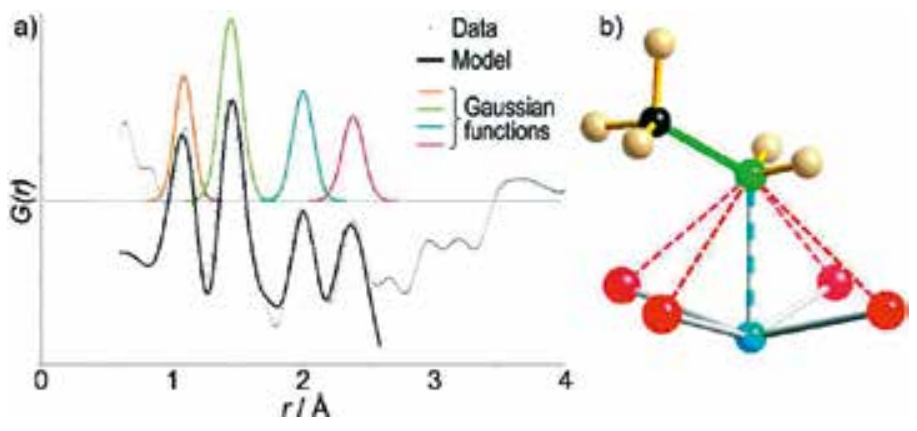

Fig. 5. d-PDF obtained after subtraction of a PDF from 'clean' $\mathrm{Al}_{2} \mathrm{O}_{3}$ from a PDF obtained from MMA-covered $\mathrm{Al}_{2} \mathrm{O}_{3}$. Reprinted with permission from Chupas et al., J. Am. Chem. Soc. 2011, 133, 8522-8524. Copyright 2011 American Chemical Society

nanoparticles are collected, and the first is subtracted from the latter either in Q- or r-space. In this way, a PDF containing only the signal from the supported nanoparticles (and possibly their interaction with the support, as in the example above) is obtained. Such d-PDF studies have been used extensively for e.g. analysis of catalyst materials ${ }^{[8 \mathrm{c}, 31]}$ and metal sorption in materials. [32]

\section{Automated Modelling of Metal Oxide Structures}

As may be clear from the examples described so far, a bottleneck in PDF analysis is identifying a suitable structural model that can be fitted to the experimental PDF. This is generally done 'manually', where a number of different models are built and tested against data. This process can limit the number of models that are tested, and can induce human bias in model selection. Banerjee et al. have recently developed an automated 'structure mining' approach for high-throughput modelling of the structure of metallic nanoparticles. ${ }^{[20 e, 33]}$ Here, thousands of structure models were automatically built and tested against the data, in order to identify which structures may be useful for further investigations. In the case of oxide materials, we recently developed a similar approach for identifying structural motifs in amorphous molybdenum oxide supported on $\mathrm{Al}_{2} \mathrm{O}_{3}$ nanoparticles. ${ }^{[34]}$ Here, we used known structures of polyoxometalates as starting point for iteratively building thousands of possible structure models. Just as many metal oxide crystal structures, polyoxometalate ions are built from edge- and corner-sharing octahedra and tetrahedra. By fitting different polyoxometalate fragments to an experimental PDF, we were able to test which types of structures and motifs gave the best fit to experimental data. ${ }^{[34]}$ Such approaches to data analysis are likely to become more important in the future, possibly also incorporating methods from data science and machine learning. ${ }^{[35]}$

\section{In situ Studies of Reactions in Materials Chemistry}

The possibility to extract atomistic structural information from PDF, regardless of long-range atomic order in the sample investigated, means that in situ PDF studies can provide unprecedented insight into atomic scale reaction mechanisms in materials. For example, PDF has allowed understanding of the formation and role of amorphous and nanostructured materials during battery cycling, ${ }^{[8 \mathrm{~d}]}$ and studies of catalytic reactions ${ }^{[36]}$ has made it possible to follow the subtle structural differences taking place during several heterogenic catalytic reactions. ${ }^{[31 a]}$

We have used PDF to gain understanding of how materials form, focusing especially on nanoparticle formation during solvothermal and hydrothermal synthesis. Solvothermal synthesis has for a long time been a preferred method for the synthesis of a range of different nanomaterials, as the method allows tuning material characteristics by exploring a large synthesis parameter space. ${ }^{[37]}$ However, the mechanisms for material formation during solvothermal synthesis remain elusive, and we are lacking an understanding of the chemical reactions driving material formation. ${ }^{[38]}$ With in situ PDF we can observe these structural changes directly. ${ }^{[39]}$ We have recently used this to study the synthesis of hexagonal tungsten oxides. ${ }^{[40]} \mathrm{We}$ were interested in the effect of solvent in the formation of tungsten oxide nanoparticles, and especially how the solvent (here water vs. oleylamine) may affect the structure of the species involved in the material formation. While it was clear from previous studies that the solvent change affected crystallite size, the influence on the reaction mechanisms itself was unknown. Fig. 6A-C show the time-resolved PDFs obtained from a hydrothermal synthesis, i.e. using water as solvent and ammonium metatungstate hydrate $\left(\left(\mathrm{NH}_{4}\right)_{6} \mathrm{H}_{2} \mathrm{~W}_{12} \mathrm{O}_{40} \cdot \mathrm{xH}_{2} \mathrm{O}\right)$ as reactant. The PDF is obtained after subtraction of the signal from the pure solvent from the data in Q-space, i.e. before the Fourier transformation. When considering the PDF from the precursor solution, we observe very clear PDF peaks, which can be fitted with the well-known $\left[\mathrm{W}_{12} \mathrm{O}_{40}\right]$ metatungstate (alpha-Keggin) cluster (Fig. 6G), which is the building block of the crystalline starting material. Upon heating, the PDF peak intensities change, and we identified tungstate $\mathrm{Y}$ ions (Fig. 6G) before the formation of crystalline hexagonal tungsten oxide nanoparticles. Fig. 6D-F show PDFs obtained from the solvothermal synthesis in oleylamine. In the precursor solution (Fig. 6F, blue), we could again identify isolated metatungstate ions, showing that even in this solvent, the crystalline starting material dissolves. However, in oleylamine, the formation of a crystalline phase from the metatungstate is completely different than in water. With heating, the structure rearranges to form a paratungstate cluster (Fig. 6G), before an amorphous phase with local structure similar to metatungstate is seen. The first crystalline phase that forms in oleylamine is the cubic pyrochlore structure $\mathrm{WO}_{3} \cdot \mathrm{H}_{2} \mathrm{O}$, which with time transforms to the hexagonal tungsten oxide. The different solvent thus completely changes not only the growth of the particles, as often discussed in literature, but also the formation mechanism itself, as it directs the structural evolution of the precursor cluster. The formation of the paratungstate cluster appears to be responsible for the formation of the pyrochlore phase, and we are currently investigating this process further under different synthesis conditions.

The study illustrates the vast possibilities PDF offers for in situ studies. With PDF, we can distinguish molecular and ionic structures in solution, ranging from large polyoxometalate ions, to smaller complexes and clusters, as well as amorphous and crystalline intermediates. The ability to study local structure, average structure, and nanostructure within one experiment opens for new possibilities for understanding chemical reactions in materials chemistry and beyond. At this point, the synthesis of a range of nanomaterials has been investigated by $\mathrm{PDF},{ }^{[41]}$ and reaction mechanisms have been observed. The next step is to fully understand how the precursor structure influences the final material in order to be able to use this knowledge in synthesis design.

\section{Outlook: What is Next for PDF Analysis?}

Over the last decades, PDF analysis has clearly become an important and established method for structure characterization of nanomaterials. The technique and data analysis methods also continue to evolve. For example, new methods in data science and machine learning are bound to have a large influence on PDF analysis (along with other data-heavy fields) in the near future. ${ }^{[42]}$ Methods from machine learning have recently been used to identify components in PDFs ${ }^{[43]}$ and determining crystallographic space group. ${ }^{[44]}$ We are currently developing machine-learning approaches to identify structural models directly from a PDF. [35] This development will help maximize the information that can be extracted from PDFs. Automated modelling, as discussed above, is also likely to get much more widely 

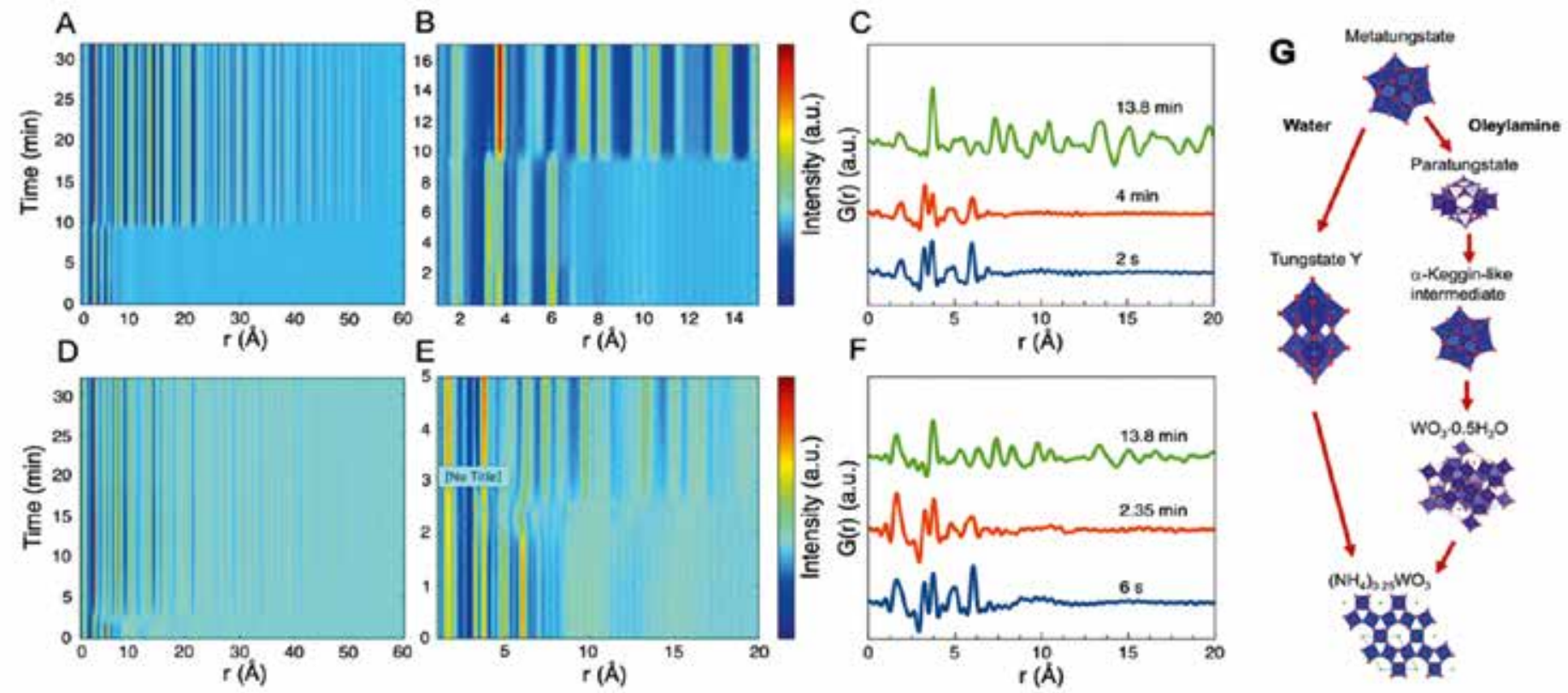

Fig. 6. (A, B) PDFs obtained from time-resolved X-ray total scattering data, collected during tungsten oxide synthesis in water. (C) PDFs obtained after $2 \mathrm{~s}, 4$ and $13.8 \mathrm{~min}$. (D, E) PDFs obtained from time-resolved X-ray total scattering data, collected during tungsten oxide synthesis in oleylamine (F) PDFs obtained after $6 \mathrm{~s}, 4 \mathrm{~min}$ and $13.8 \mathrm{~min}$. (G) The formation pathway found from the PDF analysis. Reprinted with permission from M. Juelsholt, T. Lindahl Christiansen, K. M. Ø. Jensen, J. Phys. Chem. C 2019, 123, 5110-5119. Copyright 2019 American Chemical Society.

used, ${ }^{[33,34,45]}$ and new methods and user-friendly software as well as tools for more advanced analysis continue to be developed and made available.

While the information content in the PDF is high, it is important to keep its limitations in mind: it is not always possible to determine a unique structure model from a PDF, and the uniqueness and validity of any model should always be considered. Supporting PDF models with structural information from other techniques is therefore important. In some cases, this can be done through 'complex modelling', where one structural model is refined against data from more than one technique. ${ }^{[46]}$ Such an analysis can sometimes provide more reliable models, e.g. in the combination of PDF with SAXS[47] or EXAF. [48]

As discussed above, in situ and operando PDF experiments are very useful for following chemical reactions and structural rearrangements in real time. The possibility to do position resolved PDF experiments, as is done when combining computed tomography with PDF (ctPDF) ${ }^{[49]}$ can add a completely new dimension to the understanding of real materials in devices. For example, ctPDF has been used to study position-dependent structural changes in catalysts ${ }^{[49]}$ and batteries, ${ }^{[50]}$ and this technique is likely to become much more widely applied in the future, e.g. with the development of more high energy synchrotron beamlines with microfocus beams.

Here, just a few examples of the use of PDF have been discussed, and many more can be found in the literature, where new creative and groundbreaking studies using PDF continue to appear. The immense interest in nanomaterials, and the increased focus on the properties of highly disordered materials means that the use of PDF will continue to grow, and there is no doubt that PDF is becoming a routine technique for material chemists. Just as Q-space Rietveld refinement changed the way we characterize and understand the structure of crystalline materials, PDF analysis is now making that change for nanostructured and disordered materials.

\section{Acknowledgements}

K.M.Ø.J is grateful to the Villlum Foundation for financial support through a Villum Young Investigator Grant.
[1] A. K. Cheetham, A. L. Goodwin, Nat. Mater. 2014, 13, 760, https://doi.org/10.1038/nmat4044

[2] S. J. L. Billinge, M. G. Kanatzidis, Chem. Commun. 2004, 7, 749, https://doi.org/10.1039/B309577K

[3] P. Debye, Annalen der Physik 1915, 351, 809, https://doi.org/10.1002/andp.19153510606

[4] F. Zernike, J. A. Prins, Zeitschrift für Physik A: Hadrons and Nuclei 1927, 41, 184, https://doi.org/10.1007/BF01391926

[5] P. Debye, H. Menke, Physikal. Zeit. 1930, 31, 797

[6] R. Franklin, Acta Cryst. 1950, 3, https://doi.org/10.1107/S0365110X50000264

[7] S. J. L. Billinge, P. K. Davies, T. Egami, C. R. A. Catlow, Phys. Rev. B 1991, 43, 10340, https://doi.org/10.1103/PhysRevB.43.10340

[8] a) C. A. Young, A. L. Goodwin, J. Mater. Chem. 2011, 21, 6464, https://doi.org/10.1039/C0JM04415F; b) T. Lindahl Christiansen, S. R. Cooper, K. M. Ø. Jensen, Nanoscale Adv. 2020, 13, 8725, https://doi.org/10.1039/D0NA00120A; c) P. J. Chupas, K. W. Chapman, H. Chen, C. P. Grey, Catal. Today 2009, 145, 213, https://doi.org/10.1016/j.cattod.2009.03.026; d) K. W. Chapman, MRS Bull. 2016, 41, 231, https://doi.org/10.1557/mrs.2016.26; e) S. Thakral, M. W. Terban, N. K. Thakral, R. Suryanarayanan, Adv. Drug Deliv. Rev. 2016, 100, 183, https://doi.org/10.1016/j.addr.2015.12.013

[9] a) T. Egami, S. J. L. Billinge, 'Underneath the Bragg Peaks', Vol. 7, 2nd ed., Pergamon, Oxford, 2012; b) S. J. L. Billinge, R. E. Dinnebier, 'Powder Diffraction: Theory and Practice', RSC Publishing, Cambridge, 2008.

[10] D. A. Keen, Crystallogr. Rev. 2020, 26, 143 , https://doi.org/10.1080/0889311X.2020.1797708

[11] a) P. Juhas, T. Davis, C. L. Farrow, S. J. L. Billinge, J. Appl. Cryst. 2013 46, 560, https://doi.org/10.1107/S0021889813005190; b) P. F. Peterson, M. Gutmann, T. Proffen, S. J. L. Billinge, J. Appl. Cryst. 2000, 33, 1192, https://doi.org/10.1107/S0021889800007123; c) P. Juhas, J. N. Louwen, L. van Eijck, E. T. C. Vogt, S. J. L. Billinge, J. Appl. Cryst. 2018, 51, 1492, https://doi.org/10.1107/S1600576718010002; d) A. K. Soper, 'GudrunN and GudrunX: programs for correcting raw neutron and X-ray diffraction data to differential scattering cross section', Science \& Technology Facilities Council Swindon, UK, 2011; e) B. H. Toby, R. B. Von Dreele, J. Appl. Cryst. 2013, 46, 544, https://doi.org/10.1107/S0021889813003531

[12] A. M. M. Abeykoon, C. D. Malliakas, P. Juhás, E. S. Bozin, M. G. Kanatzidis, S. J. L. Billinge, Z. Kristallogr. Cryst. Mater. 2012, 227, 248.

[13] a) J. B. Souza Junior, G. R. Schleder, F. M. Colombari, M. A. de Farias, J. Bettini, M. van Heel, R. V. Portugal, A. Fazzio, E. R. Leite, J. Phys. Chem. Lett. 2020, 11, 1564, https://doi.org/10.1021/acs.jpclett.0c00171; b) T. E. Gorelik, M. U. Schmidt, U. Kolb, S. J. L. Billinge, Microsc. Microanal. 2015, 21, 459, https://doi.org/10.1017/S1431927614014561; c) H. Shi, M. Luo, W. Wang, Comput. Phys. Commun. 2019, 238, 295, https://doi.org/10.1016/j.cpc.2018.11.019

[14] D. Keen, J. Appl. Cryst. 2001, 34, 172 , https://doi.org/10.1107/S0021889800019993 
[15] C. L. Farrow, P. Juhas, J. W. Liu, D. Bryndin, E. S. Bozin, J. Bloch, T. Proffen, S. J. L. Billinge, J. Phys. Condens. Matter 2007, 19, 335219, https://doi.org/10.1088/0953-8984/19/33/335219

[16] T.L. Christiansen, E. D. Bøjesen, M. Juelsholt, J. Etheridge, K. M. Ø. Jensen, ACS Nano 2019, 13, 8725, https://doi.org/10.1021/acsnano.9b01367

[17] D. Koziej, M. D. Rossell, B. Ludi, A. Hintennach, P. Novák, J.-D. Grunwaldt, M. Niederberger, Small 2011, 7, 377, https://doi.org/10.1002/smll.201001606

[18] L. Kihlborg, in 'Nonstoichiometric Compounds', Vol. 39, American Chemical Society, 1963, p. 37.

[19] G. Paglia, E. S. Bozin, S. J. L. Billinge, Chem. Mater. 2006, 18, 3242, https://doi.org/10.1021/cm060277j

[20] a) N. Lock, E. M. L. Jensen, J. L. Mi, A. Mamakhel, K. Noren, Q. B. Meng, B. B. Iversen, Dalton Trans. 2013, 42, 9555, https://doi.org/10.1039/C3DT00122A; b) W. Li, D. Corradini, M. Body, C. Legein, M. Salanne, J. Ma, K. W. Chapman, P. J. Chupas, A.-L. Rollet, C. Julien, K. Zhagib, M. Duttine, A. Demourgues, H. Groult, D. Dambournet, Chem. Mater. 2015, 27, 5014, https://doi.org/10.1021/acs.chemmater.5b01407; c) W. Li, M. Body, C. Legein, O. J. Borkiewicz, D. Dambournet, Inorg. Chem. 2016, 55, 7182, https://doi.org/10.1021/acs.inorgchem.6b01259; d) J.-L. Mi, K. M. Ø. Jensen, C. Tyrsted, M. Bremholm, B. B. Iversen, CrystEngComm 2015, 17, 6868, https://doi.org/10.1039/C5CE00544B; e) S. Banerjee, A. Zangiabadi, A. Mahdavi-Shakib, S. Husremovic, B. G. Frederick, K. Barmak, R. N. Austin, S. J. L. Billinge, ACS Appl. Nano Mater. 2019, 2, 6268, https://doi.org/10.1021/acsanm.9b01246

[21] a) A. L. Tiano, G. C. Papaefthymiou, C. S. Lewis, J. Han, C. Zhang, Q. Li, C. Y. Shi, A. M. M. Abeykoon, S. J. L. Billinge, E. Stach, J. Thomas, K. Guerrero, P. Munayco, J. Munayco, R. B. Scorzelli, P. Burnham, A. J. Viescas, S. S. Wong, Chem. Mater. 2015, 27, 3572, https://doi.org/10.1021/acs.chemmater.5b00767; b) F. L. Deepak, M. Bañobre-López, E. Carbó-Argibay, M. F. Cerqueira, Y. PiñeiroRedondo, J. Rivas, C. M. Thompson, S. Kamali, C. RodríguezAbreu, K. Kovnir, Y. V. Kolen'ko, J. Phys. Chem. C 2015, 119, 11947 , https://doi.org/10.1021/acs.jpcc.5b01575; c) S. R. Cooper, R. O. Candler, A. G. Cosby, D. W. Johnson, K. M. Ø. Jensen, J. E. Hutchison, ACS Nano 2020, 14, 5480, https://doi.org/10.1021/acsnano.9b09551

[22] a) F. M. Michel, L. Ehm, S. M. Antao, P. L. Lee, P. J. Chupas, G. Liu, D. R. Strongin, M. A. A. Schoonen, B. L. Phillips, J. B. Parise, Science 2007, 316, 1726, https://doi.org/10.1126/science.1142525; b) R. Harrington, D. B. Hausner, W. Xu, N. Bhandari, F. M. Michel, G. E. Brown, D. R. Strongin, J. B. Parise, Environ. Sci. Technol. 2011, 45, 9883, https://doi.org/10.1021/es2020633; c) F. M. Michel, V. Barrón, J. Torrent, M. P. Morales, C. J. Serna, J.-F. Boily, Q. Liu, A. Ambrosini, A. C. Cismasu, G. E. Brown, Proc. Natl Acad. Sci. USA 2010, 107, 2787, https://doi.org/10.1073/pnas.0910170107

[23] a) A. I. Nguyen, K. M. Van Allsburg, M. W. Terban, M. Bajdich, J. Oktawiec, J. Amtawong, M. S. Ziegler, J. P. Dombrowski, K. V. Lakshmi, W. S. Drisdell, J. Yano, S. J. L. Billinge, T. D. Tilley, Proc. Natl Acad. Sci. USA 2019, 116, 11630, https://doi.org/10.1073/pnas.1815013116; b) J. Huang, J. D. Blakemore, D. Fazi, O. Kokhan, N. D. Schley, R. H. Crabtree, G. W. Brudvig, D. M. Tiede, Phys. Chem. Chem. Phys. 2014, 16, 1814, https://doi.org/10.1039/C3CP54878C

[24] P. Du, O. Kokhan, K. W. Chapman, P. J. Chupas, D. M. Tiede, J. Am. Chem. Soc. 2012, 134, 11096, https://doi.org/10.1021/ja303826a

[25] M. W. Kanan, J. Yano, Y. Surendranath, M. Dinca, V. K. Yachandra, D. G. Nocera, J. Am. Chem. Soc. 2010, 132, 13692, https://doi.org/10.1021/ja1023767

[26] a) M. H. Han, E. Gonzalo, G. Singh, T. Rojo, Energy Environ. Sci. 2015, 8, 81, https://doi.org/10.1039/C4EE03192J; b) M. P. Browne, Z. Sofer, M. Pumera, Energy Environ. Sci. 2019, 12, 41, https://doi.org/10.1039/C8EE02495B; c) W. He, K. Ai, X. Ren, S. Wang, L. Lu, J. Mater. Chem. A 2017, 5, 19593, https://doi.org/10.1039/C7TA05076C

[27] a) V. Petkov, S. J. L. Billinge, P. Larson, S. D. Mahanti, T. Vogt, K. K. Rangan, M. G. Kanatzidis, Phys. Rev. B 2002, 65, 092105, https://doi.org/10.1103/PhysRevB.65.092105; b) D. S. Charles, M. Feygenson, K. Page, J. Neuefeind, W. Xu, X. Teng, Nat. Commun. 2017, 8, 15520, https://doi.org/10.1038/ncomms 15520 ; c) B. Song, M. Tang, E. Hu, O. J. Borkiewicz, K. M. Wiaderek, Y. Zhang, N. D. Phillip, X. Liu, Z. Shadike, C. Li, L. Song, Y.-Y. Hu, M. Chi, G. M. Veith, X.-Q. Yang, J. Liu, J. Nanda, K. Page, A. Huq, Chem. Mater. 2019, 31, 3756, https://doi.org/10.1021/acs.chemmater.9b00772; d) J. Liu, L. Yu, E. $\mathrm{Hu}$, B. S. Guiton, X.-Q. Yang, K. Page, Inorg. Chem. 2018, 57, 6873, https://doi.org/10.1021/acs.inorgchem.8b00461; e) M. S. Hvid, H. S. Jeppesen, M. Miola, P. Lamagni, R. Su, K. M. Ø. Jensen, N. Lock, IUCrJ 2019, 6, 804, https://doi.org/10.1107/S2052252519006791; f) P. Gao, P. Metz, T. Hey, Y. Gong, D. Liu, D. D. Edwards, J. Y. Howe, R. Huang, S. T. Misture, Nat. Commun. 2017, 8, 14559, https://doi.org/10.1038/ncomms14559; $\quad$ g) $\quad$ P. C. Metz, R. Koch, S. T. Misture, J. Appl. Cryst. 2018, 51, 1437, https://doi.org/10.1107/S1600576718011597
[28] A. S. Batchellor, G. Kwon, F. A. L. Laskowski, D. M. Tiede, S. W. Boettcher, J. Phys. Chem. C 2017, 121, 25421, https://doi.org/10.1021/acs.jpcc.7b10306

[29] P. J. Chupas, K. W. Chapman, G. J. Halder, J. Am. Chem. Soc. 2011, 133, 8522, https://doi.org/10.1021/ja2029574

[30] a) K. W. Chapman, P. J. Chupas, C. J. Kepert, J. Am. Chem. Soc. 2005 127, 11232, https://doi.org/10.1021/ja053266k; b) K. W. Chapman, P. J. Chupas, E. R. Maxey, J. W. Richardson, Chem. Commun. 2006, 38, 4013 , https://doi.org/10.1039/B607250J

[31] a) M. A. Newton, K. W. Chapman, D. Thompsett, P. J. Chupas, J. Am. Chem. Soc. 2012, 134, 5036, https://doi.org/10.1021/ja2114163; b) P. J. Chupas, K. W. Chapman, G. Jennings, P. L. Lee, C. P. Grey, J. Am. Chem. Soc. 2007, 129, 13822, https://doi.org/10.1021/ja076437p; c) V. Petkov, Y. Maswadeh, A. Lu, S. Shan, H. Kareem, Y. Zhao, J. Luo, C.-J. Zhong, K. Beyer, K. Chapman, ACS Appl. Mater. Interfaces 2018, 10, 10870 , https://doi.org/10.1021/acsami.7b19574

[32] a) C. M. van Genuchten, J. Pena, Environ. Sci. Proc. Impacts 2016, 18, 1030, https://doi.org/10.1039/C6EM00136J; b) W. Li, R. Harrington, Y. Tang, J. D. Kubicki, M. Aryanpour, R. J. Reeder, J. B. Parise, B. L. Phillips, Environ. Sci. Technol. 2011, 45, 9687, https://doi.org/10.1021/es200750b; c) R. Harrington, D. B. Hausner, N. Bhandari, D. R. Strongin, K. W. Chapman, P. J. Chupas, D. S. Middlemiss, C. P. Grey, J. B. Parise, Inorg. Chem. 2010, 49, 325 https://doi.org/10.1021/ic9022695; d) H.-W. Wang, D. J. Wesolowski, T. E. Proffen, L. Vlcek, W. Wang, L. F. Allard, A. I. Kolesnikov, M. Feygenson, L. M. Anovitz, R. L. Paul, J. Am. Chem. Soc. 2013, 135, 6885, https/doi.org/10.1021/ja312030e.

[33] S. Banerjee, C.-H. Liu, K. M. O. Jensen, P. Juhas, J. D. Lee, M. Tofanelli, C. J. Ackerson, C. B. Murray, S. J. L. Billinge, Acta Cryst. A 2020, 76, 24, https://doi.org/10.1107/S2053273319013214

[34] T. Lindahl Christiansen, E. T. S. Kjær, A. Kovyakh, M. L. Roderen, M. Hoj, T. Vosch, K. M. Ø. Jensen, J. Appl. Cryst. 2020, 53, 148, https://doi.org/10.1107/S1600576719016832

[35] A. S. Anker, E. T. S. Kjær, E. B. Dam, S. J. L. Billinge, K. M. Ø. Jensen, R. Selvan, ChemRxiv 2020

[36] P. J. Chupas, K. W. Chapman, H. L. Chen, C. P. Grey, Catal. Today 2009, 145, 213, https://doi.org/10.1016/j.cattod.2009.03.026

[37] K. M. Ø. Jensen, C. Tyrsted, M. Bremholm, B. B. Iversen, ChemSusChem 2014, 7, 1594, https://doi.org/10.1002/cssc.201301042

[38] S. Karthika, T. K. Radhakrishnan, P. Kalaichelvi, Cryst. Growth Des. 2016, 16, 6663, https://doi.org/10.1021/acs.cgd.6b00794

[39] E. D. Bojesen, B. B. Iversen, CrystEngComm 2016, 18, 8332, https://doi.org/10.1039/C6CE01489E

[40] M. Juelsholt, T. Lindahl Christiansen, K. M. Ø. Jensen, J. Phys. Chem. C 2019, 123, 5110, https://doi.org/10.1021/acs.jpcc.8b12395

[41] a) K. M. Ø. Jensen, H. L. Andersen, C. Tyrsted, E. D. Bøjesen, A.-C. Dippel, N. Lock, S. J. L. Billinge, B. B. Iversen, M. Christensen, ACS Nano 2014, 8, 10704, https://doi.org/10.1021/nn5044096; b) K. M. Ø. Jensen, M. Christensen, P. Juhas, C. Tyrsted, E. D. Bojesen, N. Lock, S. J. L. Billinge, B. B. Iversen, J. Am. Chem. Soc. 2012, 134, 6785 , https://doi.org/10.1021/ja300978f; c) E. D. Bojesen, K. M. Ø Jensen, C. Tyrsted, A. Mamakhel, H. L. Andersen, H. Reardon, J. Chevalier, A. C. Dippel, B. B. Iversen, Chem. Sci. 2016, 7, 6394, https://doi.org/10.1039/C6SC01580H; d) S. Birgisson, D Saha, B. B. Iversen, Cryst. Growth Des. 2018, 18, 827, https://doi.org/10.1021/acs.cgd.7b01304

[42] H. N. Bordallo, C. Lioma, J. Taylor, D. N. Argyriou, IUCrJ 2020, 7, 1, https://doi.org/10.1107/S2052252519016476

[43] a) H. S. Geddes, H. Blade, J. F. McCabe, L. P. Hughes, A. L. Goodwin, Chem. Commun. 2019, 55, 13346, https://doi.org/10.1039/C9CC06753A; b) W. Li, O. J. Borkiewicz, M. Saubanère, M.-L. Doublet, D. Flahaut, P. J. Chupas, K. W. Chapman, D. Dambournet, J. Phys. Chem. C 2018, 122, 23861, https://doi.org/10.1021/acs.jpcc.8b06573; c) K. W. Chapman, S. H. Lapidus, P. J. Chupas, J. Appl. Cryst. 2015, 48, 1619 , https://doi.org/10.1107/S1600576715016532; d) J. M. Cole, X. Cheng, M. C. Payne, Inorg. Chem. 2016, 55, 10870 , https://doi.org/10.1021/acs.inorgchem.6b00907; e) D. Olds, P. F. Peterson, M. K. Crawford, J. R. Neilson, H.-W. Wang, P. S. Whitfield, K. Page, J. Appl. Cryst. 2017, 50, 1744 , https://doi.org/10.1107/S1600576717015163

[44] a) C.-H. Liu, Y. Tao, D. Hsu, Q. Du, S. J. L. Billinge, Acta Cryst. A 2019, 75, 633, https://doi.org/10.1107/S2053273319005606; b) R. Gu, S. Banerjee, Q. Du, S. J. L. Billinge, Acta Cryst. A 2019, 75, 658, https://doi.org/10.1107/S2053273319008647

[45] a) T. Lindahl Christiansen, E. T. S. Kjær, A. Kovyakh, M. L. Röderen, M. Høj, T. Vosch, K. M.Ø. Jensen, J. Appl. Cryst. 2020, 53, 148, https://doi.org/10.1107/S1600576719016832; b) L. Yang, P. Juhas, M W. Terban, M. G. Tucker, S. J. L. Billinge, Acta Cryst. A 2020, 76, 395, https://doi.org/10.1107/S2053273320002028

[46] a) M. Eremenko, V. Krayzman, A. Gagin, I. Levin, J. Appl. Cryst. 2017, 50, 1561; b) P. Juhas, C. L. Farrow, X. Yang, K. R. Knox, S. J. L. Billinge, Acta Cryst. A 2015, 71, 562, https://doi.org/10.1107/S1600576717013140; 
c) M. J. Cliffe, M. T. Dove, D. A. Drabold, A. L. Goodwin, Phys. Rev. Lett. 2010, 104, 125501, https://doi.org/10.1103/PhysRevLett.104.125501

[47] a) A. Gagin, A. J. Allen, I. Levin, J. Appl. Cryst. 2014, 47, 619 https://doi.org/10.1107/S1600576714001046; b) D. Moscheni, F. Bertolotti, L. Piveteau, L. Protesescu, D. N. Dirin, M. V. Kovalenko, A. Cervellino, J. S. Pedersen, N. Masciocchi, A. Guagliardi, ACS Nano 2018, 12, 12558 , https://doi.org/10.1021/acsnano.8b07092; c) C. L. Farrow, C. Shi, P. Juhas, X. Peng, S. J. L. Billinge, J. Appl. Cryst. 2014, 47, 561, https://doi.org/10.1107/S1600576713034055

[48] V. Krayzman, I. Levin, M. G. Tucker, J. Appl. Cryst. 2008, 41, 705, https://doi.org/10.1107/S0021889808013277

[49] S. D. M. Jacques, M. Di Michiel, S. a. J. Kimber, X. Yang, R. J. Cernik, A. M. Beale, S. J. L. Billinge, Nat. Commun. 2013, 4, 2536, https://doi.org/10.1038/ncomms3536
[50] J. Sottmann, M. Di Michiel, H. Fjellvag, L. Malavasi, S. Margadonna, P. Vajeeston, G. B. M. Vaughan, D. S. Wragg, Angew. Chem. Int. Ed. 2017, 56, 11385, https://doi.org/10.1002/anie.201704271

\section{License and Terms}

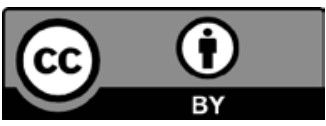

This is an Open Access article under the terms of the Creative Commons Attribution License CC BY 4.0. The material may not be used for commercial purposes.

The license is subject to the CHIMIA terms and conditions: (http:// chimia.ch/component/sppagebuilder/?view=page \&id=12).

The definitive version of this article is the electronic one that can be found at https://doi.org/10.2533/chimia.2021.368 